\title{
Nephroprotective effects of GLP-1 receptor agonists: where do we stand?
}

\author{
Charlotte M. Mosterd ${ }^{1} \cdot$ Petter Bjornstad ${ }^{2}$. Daniël H. van Raalte ${ }^{1}$
}

Received: 5 December 2019 / Accepted: 11 April 2020 / Published online: 30 April 2020

(c) The Author(s) 2020

\begin{abstract}
Glucagon-like peptide (GLP)-1 receptor agonists are the cornerstone in the treatment of hyperglycemia in many people suffering from type 2 diabetes (T2D). These drugs have potent glucose-lowering actions and, additionally, lower body weight through satiety induction while reducing blood pressure and dyslipidemia. Partly through these actions, GLP-1 receptor agonism was shown to reduce cardiovascular disease (CVD) in people with T2D with previous CVD or at high-risk thereof. In these cardiovascular safety trials, in secondary or exploratory analyses, GLP-1 receptor agonists were also shown to reduce macro-albuminuria, an accepted surrogate marker for diabetic kidney disease (DKD), a condition that still represents a major unmet medical need. In this review we will discuss the evidence which suggests renoprotection induced by GLP-1 receptor agonists and the potential mechanisms that may be involved. These include mitigation of hyperglycemia, overweight and insulin resistance, systemic and glomerular hypertension, dyslipidemia, sodium retention, inflammation and renal hypoxia. The recently initiated large-sized FLOW trial investigating the effects of semaglutide on hard renal outcomes in patients with DKD will provide clarity whether GLP-1 receptor agonists may reduce the burden of DKD in addition to their other beneficial metabolic and cardiovascular effects.
\end{abstract}

Keywords GLP-1 receptor agonists · Diabetic kidney disease · Albuminuria $\cdot$ Blood pressure $\cdot$ Incretin-based therapies

\section{Introduction}

The prevalence of people living with type 2 diabetes (T2D) has increased to over 450 million worldwide and is expected to increase further in the next decades [1]. The life expectancy of people with T2D is reduced due to increased mortality from cardiovascular disease (CVD). The strongest risk factor for CVD and mortality in T2D is diabetic kidney disease (DKD) and DKD has been proposed to account for the majority of CVD risk observed in people with diabetes. In fact, although diabetes is the main cause for end-stage kidney disease (ESKD) worldwide, many people with T2D die

Daniël H. van Raalte

d.vanraalte@amsterdamumc.nl

1 Diabetes Center, Department of Internal Medicine, Amsterdam University Medical Centers, Location VUMC, De Boelelaan 1117, 1081 HV Amsterdam, The Netherlands

2 Section of Endocrinology, Department of Pediatrics and Division of Nephrology, Department of Medicine, University of Colorado School of Medicine, Aurora, CO, USA from CVD before reaching ESKD [2]. DKD afflicts 20-40\% of people with T2D, and manifest as increased urinary albumin excretion, impaired GFR or a combination of both [3]. It is therefore clear that DKD represent a major unmet medical need. Prevention and slowing down of DKD currently focuses on risk factor mitigation, including dietary modification (e.g. low protein, salt intake and caloric targets), encouraging a healthy lifestyle with sufficient exercise and smoking cessation. Pharmacologically, lowering hyperglycemia and hyperlipidemia as well as achieving tight blood pressure control is pursued. For the latter, the renin-angiotensin-aldosterone system inhibitors (RAS) blockers are used, given their renoprotective efficacy beyond blood pressure lowering per se. Nevertheless, as demonstrated in the STENO-2 trial, residual renal and risk and overall mortality remains high, also due to the fact that the different treatment targets are often not reached [4].

Recent years have seen several pharmacological developments that may alter the outlook for people living with DKD. First, the class of sodium glucose cotransporter-2 (SGLT2) inhibitors was recently introduced. These agents lower blood glucose levels by blocking glucose reabsorption 
in the proximal tubule. Due to (temporarily) reduced sodium reuptake while continuously promoting glucosuria, these drugs induce a reduction in GFR likely via activation of tubuloglomerular feedback (TGF) that may represent reduced intraglomerular pressure [5]. In cardiovascular outcome trials (CVOTs) such as Empagliflozin Cardiovascular Outcome Event Trial in Type 2 diabetes Mellitus Patients (EMPAREG OUTCOME), CANagliflozin cardioVascular Assessment Study (CANVAS) and The Dapagliflozin Effect on Cardiovascular Events-Thrombolysis in Myocardial Infarction 58 (DECLARE-TIMI 58) [6], they reduced the onset and progression of DKD, which was confirmed in the dedicated kidney trial The Canagliflozin and Renal Endpoints in Diabetes with Established Nephropathy Clinical Evaluation (CREDENCE), which showed a canagliflozin-induced risk reduction for ESKD in people with DKD by 32\% [7]. Of note, SGLT2 inhibitors also reduce cardiovascular mortality or hospitalization for heart failure, thus showing benefits across the cardiorenal axis. Second, over a decade ago, market access was granted to the first glucagon-like peptide (GLP-1) receptor agonist. This class of drugs are frequently used by diabetologists for their strong glucose-lowering effects, beneficial safety profile as well as for their cardiovascular protection as described below. Emerging data mostly acquired from the CVOT's conducted for the various GLP-1 receptor agonists now available, also suggest that they may offer renoprotection [8]. In this review, the mechanism of action of GLP-1 receptor agonists on glucose metabolism and on the kidney are discussed as well as the (pre)clinical data that currently are suggestive of renoprotection, and potentially involved pathways.

\section{Diabetic kidney disease-a heterogenous disease with shared risk factors}

DKD, especially in people with $\mathrm{T} 2 \mathrm{D}$, is heterogenous in nature and driven by several risk factors. These include but are likely not limited to chronic hyperglycemia, hypertension, dyslipidemia, obesity, insulin resistance, sodium retention, neurohormonal activation and low-grade inflammation, all related to the metabolic syndrome $[9,10]$. In addition to these above risk factors, another driver of DKD may be glomerular hyperfiltration, i.e. increased GFR that can be manifest at the whole kidney level (GFR $>135 \mathrm{~mL} /$ $\mathrm{min} / 1.73 \mathrm{~m}^{2}$ ) in adolescents and young adults with T2D [11] or as increased single-nephron GFR in adults or older people with T2D that have reduced nephron mass; both conditions are thought to represent a state of glomerular hypertension that damages the glomerular capsule [12]. Finally, the diabetic kidney may be predisposed to chronic tissue hypoxia, in part due to impaired renal perfusion secondary to hyperglycemia-associated microvascular damage.
Additionally, the diabetic kidney has increased oxygen demand due to increased sodium reabsorption and (singlenephron) hyperfiltration via increased $\mathrm{Na}^{+}, \mathrm{K}^{+}$ATPase activity at the basolateral surface of the tubules [13]. The hyperglycemia-related increased proximal tubular sodium reabsorption results in lower sodium levels at the level of the macula densa increasing GFR via inhibiting TGF, further augmenting the amount of filtered and reabsorbed sodium with increased ATP demands. ATP generation on the other hand may be inadequate due to impaired substrate utilization due to insulin resistance and mitochondrial dysfunction [14]. The net effect of the mismatch between increased ATP demand and decreased ATP generation is increased renal oxygen consumption, ultimately rendering the kidney susceptible to renal hypoxia [15]. Hypoxia predisposes to structural defects including fibrosis and nephron loss. Analyses of renal biopsies obtained from T2D patients support a multifactorial pathophysiology as the structural damage observed varies. As such, glomerular lesions occur which include glomerular basement membrane thickening, podocyte effacement and loss, mesangial expansion, epithelial-mesenchymal transition EMT and fibrosis, collagen deposition, damage to the capillary architecture and an influx of inflammatory cells including macrophages. Beyond glomerular damage, tubular atrophy and tubulointerstitial inflammation have also been demonstrated, while vascular lesions include arterial hyalinosis [16].

As a drug class, the GLP-1 receptor agonists possess properties that could translate to improved renal energetics including its effects on substrate metabolism. It remains unclear, however, whether such effects mitigate hypoxia risk and ultimately fibrosis and nephron loss as described below.

\section{Glucagon-like peptide (GLP)-1: the incretin effect and gut-renal axis}

GLP-1 was discovered to be one of the key hormones to underlie the so-called incretin effect, i.e. an augmented insulin response to an oral glucose load compared with intravenous (IV) glucose administration. GLP-1 is secreted from $L$ cells in the small intestine upon food stimuli including carbohydrates, lipids, proteins, amino acids, bile acids and short-chain fatty acids. GLP-1 has a short half-life of $\sim 2-3$ min due to rapid inactivation by the enzyme dipeptidyl peptidase (DPP)-4. Through neural pathways as well as via receptor-mediated effects, GLP-1 reduces postprandial hyperglycemia by stimulation of insulin secretion, suppression of glucagon release, inhibition of gastric emptying and small intestinal peristalsis, and induction of satiety which limits meal size [17]. The glucometabolic effects of GLP-1 have been reviewed extensively elsewhere [18] and are not the focus of the current review. 
In addition to its glucoregulatory effects, GLP-1 was shown to interact with other organs as well, which is not surprising given the widespread expression of GLP-1 receptors in the human body. As such, GLP-1 has been linked to the kidney as a feed-forward sensor that is part of a socalled gut-renal axis [19]. In non-diabetic mice, GLP-1 infusion increased GFR by reducing preglomerular resistance [20], an observation that could potentially explain the physiological increase in GFR observed in the postprandial compared to the fasting state, although human studies infusing GLP-1 peptide could not replicate this observation [21]. Meal-induced increments in GFR could serve to enhance the elimination of nitrogen waste products as well as electrolytes such sodium and potassium. Indeed, both in rodents and humans, GLP-1 infusion increased fractional sodium excretion [20], which has been linked to the finding that sodium is more rapidly excreted following an oral versus equivalent IV sodium load in a number of experimental studies in healthy individuals, independent of factors such as atrial natriuretic peptide (ANP) and aldosterone [22]. GLP-1 may induce sodium excretion by blocking sodium reabsorption in the proximal tubule through inhibition of sodium-hydrogen exchanger isoform 3 (NHE3) activity [23], as no GLP-1 receptors appear to be present in human kidneys outside of the renal vasculature [19].

In people with $\mathrm{T} 2 \mathrm{D}$, the incretin effect is severely attenuated, despite similar levels of circulating GLP-1. However, a dose-dependent insulinotropic and glucagonostatic response is preserved in response to exogenous GLP-1 treatment and 6-week subcutaneous GLP-1 infusion was shown to improve $\mathrm{HbA1c}$ levels by $1.3 \%$ [18]. However, due to its indicated short half-live, GLP-1 peptide is an unattractive therapeutic agent on itself, which stimulated the quest for GLP-1 based compounds with better bioavailability.

\section{Development of GLP-1 receptor agonists}

The most important strategy to develop compounds with extended in vivo half-life of GLP-1 was by making DPP-4 resistant GLP-1 receptor agonists. Over the last decade and a half, several (injectable and most recent an oral) GLP-1 receptor agonist formulations have been introduced for the management of hyperglycemia in people with T2D [24].

The first molecules that were developed were based on an exendin- 4 backbone which was isolated from the saliva of the Gila monster (Heloderma suspectum) which shares 53\% homology with GLP-1. Exenatide (twice-daily) and lixisenatide are GLP-1 receptor agonists based on exendin-4 that overcome cleavage by DPP-4, however, their half remains short with $\sim 2-4$ h due to renal elimination. They are classified as short-acting or postprandial GLP-1 receptor agonists due to their glucose-lowering effects in the postprandial state
[24]. Second generation GLP-1 receptor agonists that were developed have more similarity with human GLP-1 and are linked to larger carrier molecules to limit renal elimination. These include the once-daily GLP-1 receptor agonist liraglutide and the once-weekly compounds albiglutide, dulaglutide, semaglutide and a long-acting release formulation of exenatide (formulated within biodegradable polymeric microspheres). The long-acting GLP-1 receptor agonists have strong effects on insulin secretion and fasting glucose levels, but lose their postprandial glucose-lowering properties due to receptor tachyphylaxis. GLP-1 receptor agonists lower $\mathrm{HbA} 1 \mathrm{c}$ by $1-1.5 \%$ but this may be dependent of the type of GLP-1 receptor agonist prescribed, the dosage, baseline HbAlc levels and glucose-lowering co-medication [24]. Due to their glucose-lowering efficacy as well as other beneficial effects on blood pressure, body weight and lipid metabolism, they have taken a central place in the management of hyperglycemia in people with T2D. Based on studies in people with chronic kidney disease (CKD), GLP-1 receptor agonists were shown to be safe (no increased risk for acute kidney injury (AKI) was observed) and efficacious also at lower eGFR ranges [25]. Liraglutide is currently approved for people with T2D and an eGFR $>15 \mathrm{~mL} /$ $\min / 1.73 \mathrm{~m}^{2}$, and besides metformin and insulin is the only diabetes medication approved by FDA for adolescents with youth-onset T2D [26].

\section{GLP-1 receptor agonists and renal outcomes}

Based on the US FDA industry guidance issued in 2008, all new glucose-lowering agents were required to perform a large-sized placebo-controlled cardiovascular outcome/ safety trial, usually conducted in people with T2D with pre-existent or at high-risk of developing CVD. At present, seven trials have currently been reported for GLP-1 receptor agonists which include ELIXA (Evaluation of LIXisenatide in Acute coronary syndrome; lixisenatide), LEADER (Liraglutide Effects and Action in Diabetes: Evaluation of cardiovascular outcome Results; liraglutide), SUSTAIN-6 (Trial to Evaluate Cardiovascular and Other Long-term Outcomes With Semaglutide in Subjects With Type 2 Diabetes; subcutaneous semaglutide), EXSCEL (EXenatide Study of Cardiovascular Event Lowering; exenatide once weekly), REWIND (Researching Cardiovascular Events With a Weekly Incretin in Diabetes; dulaglutide), HARMONY (Albiglutide and cardiovascular outcomes in patients with T2D and cardiovascular disease; albiglutide) and PIONEER-6 (oral semaglutide) [8]. The primary outcomes of these trials were 3 -point major adverse cardiovascular event (3-MACE) and the studies have together provided a wealth of data that, amongst others, have changed clinical guidelines. We refer to a recent 
systematic review and meta-analysis for details regarding the effects of GLP-1 receptor agonists on cardiovascular outcomes [8]. In short, despite some heterogeneity across the trials, GLP-1 receptor agonists were shown to reduce the hazard ratio's [95\% CI] for 3-MACE by 12 [6-18]\%, cardiovascular death by 12 [4-19]\%, myocardial infarction by $9[0-16] \%$ and stroke by $16\{7-24] \%$. In these trials, data on renal outcomes were also collected (Table 1), although not all trials had this as a prespecified outcome, and therefore the data should be interpreted with caution.

In ELIXA, the effects of lixisenatide versus placebo in 6,026 people with T2D and a recent acute coronary event were investigated. Lixisenatide reduced urinary albumin-tocreatinine ratio (UACR) versus placebo at 108 weeks of follow-up by $10 \%(\mathrm{p}=0.004)$. Correction for small differences in glycemic control during the trial diminished significance indicating some glucose-dependency [27]. In a separate analysis, lixisenatide reduced incident macroalbuminuria, while adjustments for baseline characteristics such as age, blood pressure and eGFR, as well as on trial HbA1c, blood pressure and weight, did not significantly attenuate the association [28]. In a sub-analysis in people with albuminuria at baseline, lixisenatide reduced UACR by $21 \%$ (in people with microalbuminuria) $39 \%$ (in people with macroalbuminuria). Lixisenatide did not change eGFR decline, and incidences of hard renal endpoints were too low to be meaningfully analyzed [28].

Subsequently, LEADER and SUSTAIN-6 were reported, which investigated respectively liraglutide and semaglutide vs. placebo in 9340 and 3297 people with T2D and high cardiovascular risk. LEADER [29] and SUSTAIN-6 [30] reported a prespecified renal composite of new onset or persistent macroalbuminuria, persistent doubling of serum creatinine and eGFR $\leq 45 \mathrm{~mL} / \mathrm{min} / 1.73 \mathrm{~m}^{2}$, need for renal replacement therapy (RRT), and renal death. Liraglutide reduced this renal outcome by $22 \%$ after 3.8 years follow up, whereas semaglutide showed a reduction of $36 \%$ after 2.1 years. Importantly, these reductions were solely driven by reductions in macroalbuminuria without effects on hard renal endpoints. As liraglutide and semaglutide induced stronger $\mathrm{HbA} 1 \mathrm{c}$ reductions versus placebo then lixisenatide, it was important that the authors showed in reply to a letter to the editor that the anti-albuminuric effects were independent of glucose-lowering in post-hoc analyses. Regarding eGFR trajectories, both drugs induced a slightly slower decline in eGFR compared to the placebo groups, which was more pronounced in people with moderate (eGFR $30-59 \mathrm{~mL} / \mathrm{min} / 1.73 \mathrm{~m}^{2}$ ) or severe (eGFR $<30 \mathrm{~mL} /$ $\mathrm{min} / 1.73 \mathrm{~m}^{2}$ ) impaired renal function at baseline. In contrast to RAAS blockers and SGLT2 inhibitors however, this higher eGFR at end of treatment was not caused by an initial drop in eGFR with stabilization over time (suggestive of a reduction in glomerular pressure), but rather by an upsurge in eGFR with similar rates of eGFR decline over time versus placebo $[29,30]$.

In the EXSCEL trial, 14,752 people with T2D and previous CVD were exposed to exenatide once weekly or placebo for a median duration of 3.2 years. A prespecified renal endpoint which was driven by incident macroalbuminuria was significantly reduced after adjustments for baseline factors including age, sex, BMI, HbA1c and eGFR. No other endpoints were affected, including eGFR (Table 1). [31].

While the HARMONY outcomes reported no change in eGFR after 16 months of treatment, no other renal outcomes were presented, which also holds true for the recently completed PIONEER-6 trial with oral semaglutide.

Finally, the REWIND trial reported renal outcomes after 5.4 years of dulaglutide treatment versus placebo. In addition to reducing albuminuria, dulaglutide reduced the surrogate renal endpoints of $40 \%$ or $50 \%$ decline in eGFR (HR 0.70 and 0.56 respectively; $\mathrm{p}<0.001$ for both). The absolute eGFR differences between the groups by the end of the study were small $\left(0.42 \mathrm{ml} / \mathrm{min} / 1.73 \mathrm{~m}^{2}\right)$ and the eGFR slopes did not meaningfully differ after the initial weeks of treatment [32].

\section{GLP-1 receptor agonist treatment in people with DKD}

Limitations of the above-described studies include exploratory renal endpoints with limited adjudication, and small number of participants with baseline DKD. Currently, there are only a few trials that investigated the effects of a GLP-1 receptor agonist in people with DKD, however they focused primarily on glucose-lowering and two were of short duration. In the AWARD-7 trial, the effects of low- and highdose dulaglutide versus titrated insulin glargine, both added to prandial insulin humulin, was compared in 576 people with T2D and moderate to severe CKD. Compared to insulin glargine, dulaglutide reduced UACR by 39 (10-69) \% in patients with macroalbuminuria at baseline [33]. In addition, dulaglutide lead to lower eGFR decline compared with titrated insulin glargine: the cystatin C-based eGFR decline of 52-week dulaglutide high-dose was $0.7 \mathrm{~mL} / \mathrm{min} / 1.73 \mathrm{~m}^{2}$ and low-dose $0.7 \mathrm{~mL} / \mathrm{min} / 1.73 \mathrm{~m}^{2}$ vs. $3.3 \mathrm{~mL} / \mathrm{min} / 1.73 \mathrm{~m}^{2}$ with insulin glargine. Similar to what was observed in the participants with CKD stage 3-4 in LEADER, the differences in eGFR were caused by an initial upsurge in eGFR with similar decline rates over the course of the study. In LiraRenal (liraglutide versus placebo in people with type 2 diabetes and eGFR $30-59 \mathrm{~mL} / \mathrm{min} / 1.73 \mathrm{~m}^{2}$ ), liraglutide effective lowered $\mathrm{HbA1c}$ and body weight, but did not affect eGFR after 26 weeks of treatment [25]. Similarly, in PIONEER-5 where 324 patients with diabetes and eGFR $30-59 \mathrm{~mL} / \mathrm{min} / 1.73 \mathrm{~m}^{2}$, oral semaglutide showed safety 
Table 1 Renal outcomes in six major cardiovascular safety trials assessing glucagon-like peptide-1 receptor agonists in people with type 2 diabetes



†Significant versus insulin glargine $(p<0.05)$.

††Significant versus placebo $(p<0.05)$

${ }^{*}$ Composite renal outcome, new -onset persistent macroalbuminuria, persistent doubling of serum creatinine and eGFR $<45$

$\mathrm{mL} / \mathrm{min}$ per $1.73 \mathrm{~m}^{2}$, requirement for kidney replacement therapy or kidney disease death in number of patients (rate per 1000 patient-years of observation).

${ }^{\star *}$ number per 1000 patients-years of observation. ${ }^{* * *}$ number per 100 patients-years of observation.

Abbreviations: $\mathrm{BMI}=$ body mass index. $\mathrm{BP}=$ blood pressure. eGFR=estimated glomerular filtration rate. $\mathrm{HF}=$ heart failure. $\mathrm{NR}=$ not reported. $\mathrm{SBP}=$ systolic blood pres sure. $\mathrm{UACR}=$ urine albumin-to-creatinine ratio 
and efficacy on glycemic endpoints, but did not alter eGFR, although it lowered UACR to a similar extent as the injectable GLP-1 receptor agonists [34].

\section{Potential mechanisms involved in albuminuria lowering}

Several mechanisms have been proposed to explain how GLP-1 receptor agonists may lower albuminuria. These involve classical renal risk factors as well as some novel mediators of DKD. We will address these risk factors/pathways individually below (summarized in Fig. 1). We have started with 'classic renal risk factors' and then move on to more novel/experimental pathways.

\section{Hyperglycemia}

Observational studies have clearly showed a causal link between hyperglycemia and DKD incidence, mechanistically caused hyperglycemia-induced glomerular hyperfiltration as well pathways associated with renal glucose toxicity [9]. Long-term intervention trials demonstrated that intensive glucose-lowering versus lenient glucose control reduced DKD incidence. In the United Kingdom Prospective Diabetes study (UKPDS) intensive glucose control reduced the risk of macroalbuminuria by $33 \%$. Similarly, in The Action in Diabetes and Vascular Disease: Preterax and Diamicron MR Controlled Evaluation (ADVANCE) trial, intensive glucose control reduced ESKD by 46 (95\% CI 15-66)\% [35]. Thus, given the potent glucose-lowering actions of the GLP-1 receptor agonists as described (HbA1c reduction $1-1.5 \%$ and even more pronounced for the most recent long-acting GLP-1 receptor agonists), reduction in hyperglycemia may contribute to their strong anti-albuminuric effects. However, post hoc analyses showed only mild changes in the effect size when adjusted for on-trial HbA1c concentrations suggesting additional mechanisms independent of glycemic control.

\section{Hypertension}

Reducing blood pressure has proven to be renoprotective, particularly demonstrated in studies involving RAS blockers [36, 37], although exact blood pressure targets remain debated. Thus, a glucose-lowering agent that also reduces

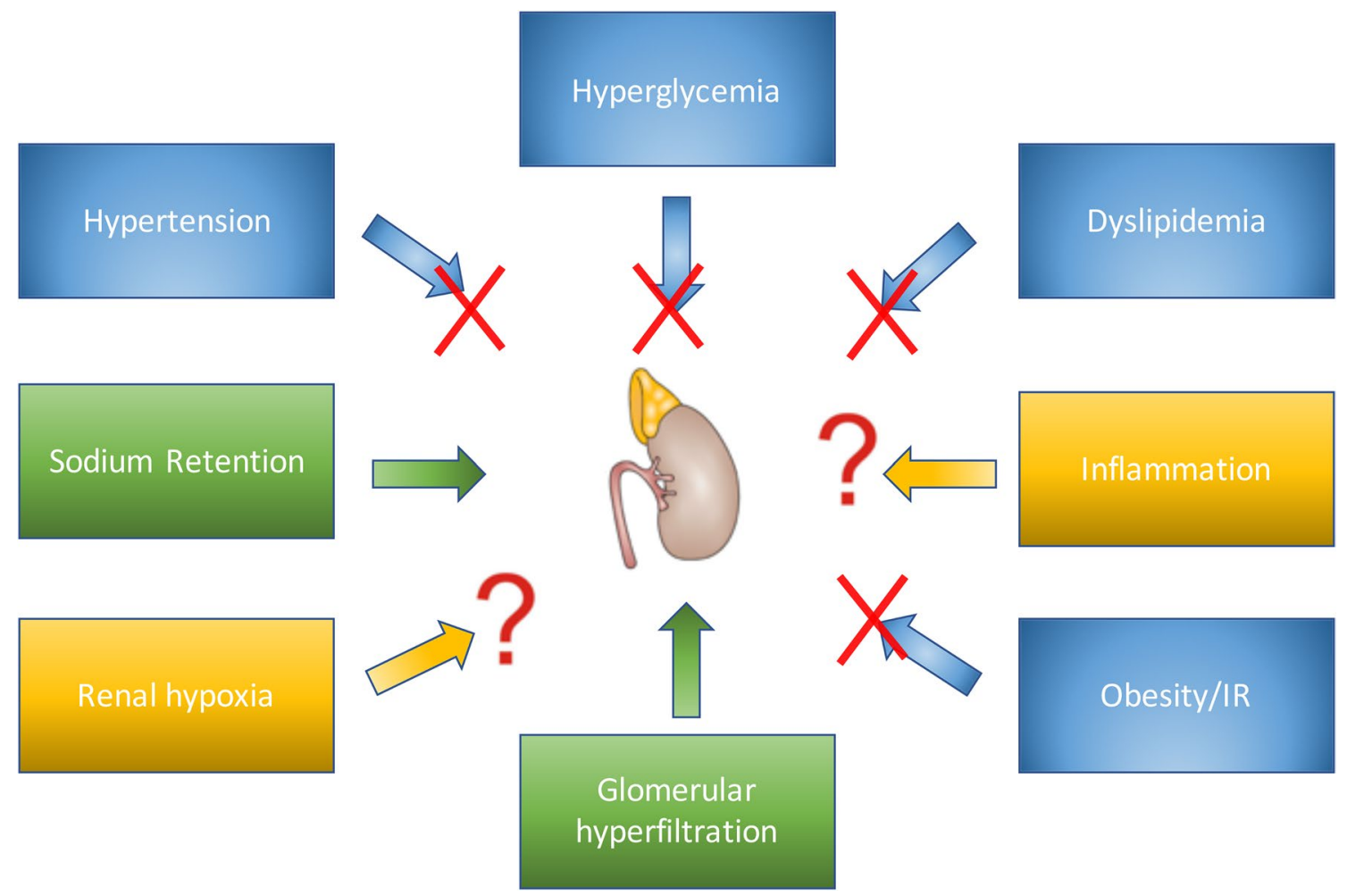

Fig. 1 GLP-1 receptor agonists effects on established and presumed drivers of diabetic kidney disease (DKD). In blue boxes, established renal risk factors that are mitigated by GLP-1 receptor agonism (illustrated by a red cross). The risk factors of sodium retention and glomerular hyperfiltration (green boxes) are likely not reduced by GLP-1 receptor agonists. Finally, in yellow boxes, more recently discovered mechanisms of renal damage including inflammation and renal hypoxia are indicated. It is unclear whether GLP-1 receptor agonism modulates these pathways (color figure online) 
blood pressure is of added benefit. Mechanistically, systemic hypertension may drive DKD by inducing pressure-induced glomerular damage. GLP-1 receptor agonists increase blood pressure acutely, but lower blood pressure after prolonged treatment [19]. The systolic blood pressure reduction is 3-4 mmHg with slight differences observed between the different GLP-1 receptor agonists. The mechanisms by which GLP-1 receptor agonists reduce blood pressure are incompletely understood, but may relate to improvements in insulin sensitivity secondary to weight loss. In addition, inhibition of RAAS activity, improved endothelial function and augmented natriuresis have been proposed. Similar to hyperglycemia, however, controlling for on-trial blood pressure did not significantly alter the reductions in albuminuria induced by GLP-1 receptor agonist treatment in the trials [28].

\section{Dyslipidemia}

Dyslipidemia is a strong risk factor for both CVD and DKD, both with respect to elevated LDL cholesterol, triglycerides and reduced HDL concentrations. Biopsy studies in animals indicated that hyperlipidemia may cause glomerular lipid depositions, an observation that was termed glomerular atherosclerosis [38]. Data from lipid-lowering trials, most notably conduced with statin therapy, were however unable to demonstrate benefits of LDL-lowering on renal outcomes $[39,40]$. GLP-1 receptor agonist therapy was shown in a meta-analysis to induce small reductions in the levels of LDL cholesterol, total cholesterol and triglycerides, but not to improve HDL cholesterol levels in comparison to placebo and active comparators [19]. Mechanistically, GLP-1 receptor agonists reduce intestinal chylomicron production and secretion, in addition to potential inhibit on hepatic very-low density lipoprotein (VLDL) production [41]. It is uncertain whether these actions contribute to potential renoprotective effects of GLP-1 receptor agonists.

\section{Obesity and insulin resistance}

Obesity and associated insulin resistance play a crucial role in the development of nearly all metabolic abnormalities associated with T2D. With respect to the kidneys, obesity may predispose to albuminuria and impaired GFR due to the fact that it contributes to many of the established renal risk factors summarized above. Additionally, obesity has been linked to DKD by provoking glomerular hyperfiltration, secondary to changes in sodium retention and increased sympathetic nervous system activity [42]. Intrarenal fat deposition ('fatty kidney'), in particular fat accumulation in the renal sinus (RSF), has been linked to hypertension [43], renal inflammation and fibrosis, and unfavorable renal hemodynamic function [44]. Intervention trials aimed at weight loss have indeed showed evidence for renoprotection. As such, $4 \mathrm{~kg}$ weight loss observed in the landmark life style study LOOK-AHEAD reduced the incidence of very-high-risk chronic kidney disease by $31 \%$ at 8 years of follow-up [45], and results from bariatric surgery cohorts have similarly observed renal benefits (reduced hyperfiltration, reduction in UACR) of weight loss [46]. In a recent comparison analysis of the Teen-LABS and TODAY studies, bariatric surgery was shown to attenuate DKD to a substantially greater degree than standard medical therapy in severely obese youth and young adults with T2D [47]. It should be noted, however, that estimated eGFR may be biased in this population where severe weight loss may be accompanied by muscle mass loss, making eGFR that was often reported less reliable. GLP-1 receptor agonist therapy induces weight loss of up to $2 \mathrm{~kg}$ compared with placebo and up to $7 \mathrm{~kg}$ compared with antihyperglycemic drug classes that are associated with weight gain, most notably insulin therapy [19]. It should be noted that there is much variation with respect to individual responses and the different GLP-1 receptor agonists, with the long-acting compounds inducing stronger body weight reductions. Nevertheless, correction for weight loss in the CVOT's and AWARD-7 study only partly explained the observed albuminuria reduction. This BMI-correction does not rule out a role for reducing local fat deposition, either in close proximity to kidney tissue or located in the perivascular space. In preclinical studies, GLP-1 receptor treatment was able to reduce renal fat content and concomitantly reduce inflammation and formation of reactive oxygen species (ROS) [48]. However, such mechanisms still need to be interrogated further in clinical research.

\section{Sodium retention}

Excessive salt intake, characteristic of a Western diet, has been linked to hypertension, CVD, and CKD [49]. This may be particularly true for people with T2D who have greater salt sensitivity, secondary to impaired nonosmotic sodium storage or impaired sodium excretion [50]. The latter is associated with obesity, insulin resistance and exogenous insulin therapy that may drive tubular reabsorption of sodium. Similar to endogenous GLP-1 activity, GLP-1 receptor agonists affect tubular function, as intravenous administration of exenatide induced natriuresis in healthy males and individuals with T2D [51, 52]. The most likely involved tubular transporter mediating the natriuretic effects of GLP-1 is NHE3 which is located at the luminal border of the proximal tubule. Indeed, GLP-1 receptor agonists phosphorylate NHE3 at the protein kinase A consensus sites Ser552 and Ser605, thereby reducing its activity [53]. Acute GLP-1 receptor agonist treatment also enhances renal lithium clearance 
(a marker of proximal tubular sodium reabsorption) and urinary $\mathrm{pH}$ making it plausible that NHE3 transporter in the proximal tubule is indeed involved (reference). After prolonged administration, however, this natriuretic effect disappears as the kidney quickly adjust tubular sodium transport to match sodium excretion to sodium intake [54]. If enhanced sodium excretion would contribute to the cardiovascular and renal effects of GLP-1 receptor agonists, one would expect a reduction in plasma volume (or as a proxy: increased hematocrit) [55] or total body sodium content [56]. This seems however not to be the case and makes the role for altered sodium homeostasis unlikely to account for the renal and cardiovascular effects of GLP 1 receptor agonism.

\section{Hyperfiltration}

Glomerular hyperfiltration is increasingly recognized as a risk factor that drives the progression of DKD. This can present either as elevated whole-kidney GFR in people with preserved nephron mass (e.g. in young individuals with early disease) as well as single-nephron hyperfiltration in older people with reduced nephron mass and/or later stages of disease [57]. Drugs that alter glomerular hyperfiltration such as RAS blockers and SGLT-2 inhibitors $[58,59]$ are among the most powerful drugs to prevent and reduce DKD burden. Given the effects of GLP-1 receptor agonists on proximal tubular sodium handling, in analogy with SGLT2 inhibitors, they could potentially alter renal hemodynamics by activating TGF. An early study by Gutzwiller et al. in hyperfiltering patients few of which had diabetes [60] as well as an open-label, uncontrolled study with liraglutide [61] suggested reductions in (estimated) GFR derived from creatinine clearance and ${ }^{51} \mathrm{Cr}$-EDTA, respectively. Several subsequent studies have more comprehensively detailed the effects of GLP-1 receptor agonism on glomerular hemodynamics using state-of-the-art inulin and para-aminohippuric acid (PAH) clearance techniques to measure GFR and effective renal plasma flow (ERPF), respectively. In contrast to the results of earlier studies, exenatide infusion increased GFR, ERPF and estimated glomerular hydraulic pressure in overweight but otherwise healthy men [51]. This increment in GFR and ERPF seemed to be caused by a reduction in estimated afferent renal arteriolar resistance, which was partly dependent on nitric oxide (NO). Subsequent studies with similar design showed no effect of acute or prolonged exenatide or liraglutide treatment on renal hemodynamics in people with T2D with or without DKD [52-54].

Thus, at present there is insufficient evidence to support attenuation of glomerular hyperfiltration in response to GLP-1 receptor agonists, as seems the case for RAS blockers or SGLT2 inhibitors.

\section{Inflammation}

In recent years, the role of the immune system in the pathogenesis of DKD has received much attention. Kidney biopsies derived from people with DKD have shown that different cells of the immune system, most notably classically-activated macrophages and T-cells, accumulate in the kidney and interact with resident kidney cells [62]. In addition, both centrally and locally produced pro-inflammatory cytokines and chemokines, including tumor necrosis factor (TNF)-alpha, monocyte chemoattractant protein-1 (MCP-1/ CCL2), various interleukins (IL-6 and IL-1 $\beta$ ) as well as other cytokines under control of the master regulator nuclear factor kappa beta (NF- $\kappa \beta$ ) are increased in people with DKD. Increasing evidence shows that both pro-inflammatory and anti-inflammatory stimuli modulate GLP-1 secretion, while GLP-1 in turn regulates the immune system, both at the level of the kidneys and blood vessels [63]. In several preclinical studies, GLP-1 receptor agonists lowered the systemic levels of the pro-inflammatory cytokines, inhibited pro-inflammatory signaling pathways independent of glucose-lowering while concomitantly improving histological features of DKD [19]. This reduction in inflammatory tone could certainly contribute to improved renal outcomes and has also been speculated to underlie the cardiovascular benefits of GLP-1 receptor agonists. However, clinical studies are needed to elucidate these potential inflammatory pathways in response to GLP-1 receptor agonists.

\section{Renal hypoxia}

Finally, renal hypoxia has been proposed to be a unifying pathway for most etiologies of CKD, including DKD [15]. People with DKD are prone to chronic hypoxia due to the combination of reduced oxygen supply through microvascular damage, excessive renal energy expenditure due to hyperglycemia-related glomerular hyperfiltration and associated increased sodium reabsorption, and a less oxygen efficient fuel profile. The less oxygen-efficient fuel profile observed in people with T2D is thought to be secondary to insulin resistance and mitochondrial dysfunction altering substrate metabolism as indicated previously. Based on their effects on endothelial function, GLP-1 receptors could improve renal vascularization and oxygen supply, whilst lowering oxygen consumption by attenuating in obesity-related hyperfiltration. On the other end of the equation, GLP-1 induced changes in substrate metabolism through improvements in insulin sensitivity and mitochondrial dysfunction could enhance ATP production, as was suggested in a study in rodents [48]. It is currently unclear whether GLP-1 receptor agonists modulate oxygen physiology in humans and whether they could ameliorate renal hypoxia. Mechanistic trials are needed to evaluate whether 
GLP-1 receptor agonists can correct the potential metabolic imbalance between increased renal energy expenditure and impaired substrate metabolism proposed to underlie DKD.

\section{Conclusion and future perspectives}

In recent years, GLP-1 receptor agonists have received a central position in the management of hyperglycemia in people with T2D given their potent glucose-lowering actions. In addition, they improve blood pressure, body weight and dyslipidemia and are consistently shown to reduce CVD in a high-risk T2D population. The GLP-1 receptor agonists are safe to use in people with DKD, however, whether they are truly nephroprotective remains to be seen. Trials have shown that GLP-1 receptor agonists lower albuminuria, however it is uncertain whether this will translate into improvements in hard renal outcomes. Similarly, the small changes in eGFR trajectories (i.e. subtle reductions in eGFR decline versus placebo or titrated insulin) do not necessarily indicate renoprotection. As the GLP-1 receptor agonists induce an initial upsurge in eGFR with similar trajectories over time, this pattern may not indicate a reduction of glomerular pressure. The answers to these outstanding gaps in knowledge are currently addressed in the FLOW trial (Semaglutide on the Progression of Renal Impairment in Subjects With Type 2 Diabetes and Chronic Kidney Disease; NCT03819153). In this trial, the effects of once weekly subcutaneous semaglutide in patients with macro-albuminuria and impaired eGFR are examined over 5 years of follow-up. The primary endpoint is a composite of persistent eGFR decline of $>50 \%$, reaching ESKD, death from kidney disease or death from CVD. Given the recent results of the CREDENCE trial, many patients will also be treated with an SGLT2-inhibitor, allowing the trial to study the combined use of these agents on renal outcomes. Of note, in people with advanced DKD, additional glucose-lowering beyond SGLT2 inhibition will be necessary, making GLP-1 receptor agonists valuable drugs in this population. Finally, the FLOW trial may shed further light on the potential mechanisms involved on how GLP-1 receptor agonism could alter renal physiology, combined with future mechanistic studies that aim to find proof for the effects of GLP-1 receptor agonists on the renal risk factors/pathophysiological mechanisms related to DKD as summarized in this review.

Disclosures PB has acted as a consultant for Bayer, Bristol-Myers Squibb, Boehringer Ingelheim, Sanofi, Novo Nordisk and Horizon Pharma. PB serves on the advisory board of XORTX. DHvR has acted as a consultant and received honoraria from Boehringer Ingelheim and Eli Lilly, MSD, Novo Nordisk, and Sanofi and has received research operating funds from Boehringer Ingelheim-Eli Lilly Diabetes Alliance, MSD, AstraZeneca, and Novo Nordisk.
Author contributions The authors were fully responsible for all content and editorial decisions and were involved at all stages of manuscript development and have approved the final version.

Funding DHvR is supported by a research fellowship from the Dutch Diabetes Foundation and EU Marie Curie program. PB receives salary and research support by NIH/NIDDK (Grant No. K23 DK116720-01), in addition to research support by Juvenile Diabetes Research Foundation (JDRF), NIDDK/DiaComp, NIDDK/KPMP, Thrasher Research Fund, International Society of Pediatric and Adolescent Diabetes (ISPAD), Colorado Clinical \& Translational Sciences Institute (CCTSI) and Center for Women's Health Research at University of Colorado.

\section{Compliance with ethical standards}

Conflict of interest Charlotte M. Mosterd has no conflict of interest. Petter Bjornstad has acted as a consultant for Bayer, Bristol-Myers Squibb, Boehringer Ingelheim, Sanofi, Novo Nordisk and Horizon Pharma. Petter Bjornstad also serves on the advisory board of XORTX. Daniel H. van Raalte. has acted as a consultant and received honoraria from Boehringer Ingelheim, Eli Lilly, Merck, Novo Nordisk and Sanofi and has received research operating funds from the Boehringer Ingelheim-Eli Lilly Diabetes Alliance, MSD, AstraZeneca and Novo Nordisk.

Ethical statement This article does not contain any studies with human participants performed by any of the authors.

Open Access This article is licensed under a Creative Commons Attribution 4.0 International License, which permits use, sharing, adaptation, distribution and reproduction in any medium or format, as long as you give appropriate credit to the original author(s) and the source, provide a link to the Creative Commons licence, and indicate if changes were made. The images or other third party material in this article are included in the article's Creative Commons licence, unless indicated otherwise in a credit line to the material. If material is not included in the article's Creative Commons licence and your intended use is not permitted by statutory regulation or exceeds the permitted use, you will need to obtain permission directly from the copyright holder. To view a copy of this licence, visit http://creativecommons.org/licenses/by/4.0/.

\section{References}

1. Cho NH, Shaw JE, Karuranga S, Huang Y, da Rocha Fernandes JD, Ohlrogge AW et al (2018) IDF Diabetes Atlas: global estimates of diabetes prevalence for 2017 and projections for 2045 . Diabetes Res Clin Pract 138:271-281

2. Gansevoort RT, Correa-Rotter R, Hemmelgarn BR, Jafar TH, Heerspink HJL, Mann JF et al (2013) Chronic kidney disease and cardiovascular risk: epidemiology, mechanisms, and prevention. Lancet 382:339-352

3. Thomas MC, Cooper ME, Zimmet P (2016) Changing epidemiology of type 2 diabetes mellitus and associated chronic kidney disease. Nat Rev Nephrol 12(2):73-81

4. Muskiet MHA, Tonneijck L, Smits MM, Kramer MHH, Heerspink HJL, van Raalte DH (2015) Pleiotropic effects of type 2 diabetes management strategies on renal risk factors. Lancet Diabetes Endocrinol 3(5):367-381

5. van Bommel EJM, Muskiet MHA, Tonneijck L, Kramer MHH, Nieuwdorp M, van Raalte DH (2017) SGLT2 inhibition in the 
diabetic kidney - from mechanisms to clinical outcome. Clin J Am Soc Nephrol 12:700-710

6. Zelniker TA, Wiviott SD, Raz I, Im K, Goodrich EL, Furtado RHM et al (2019) Comparison of the effects of glucagon-like peptide receptor agonists and sodium-glucose cotransporter 2 inhibitors for prevention of major adverse cardiovascular and renal outcomes in type 2 diabetes mellitus: systematic review and metaanalysis of cardio. Circulation 139:2022-2031

7. Perkovic V, Jardine MJ, Neal B, Bompoint S, Heerspink HJL, Charytan DM et al (2019) Canagliflozin and renal outcomes in type 2 diabetes and nephropathy. N Engl J Med 380(24):2295-2306

8. Kristensen SL, Rørth R, Jhund PS, Docherty KF, Sattar N, Preiss $\mathrm{D}$ et al (2019) Cardiovascular, mortality, and kidney outcomes with GLP-1 receptor agonists in patients with type 2 diabetes: a systematic review and meta-analysis of cardiovascular outcome trials. Lancet Diabetes Endocrinol 7(10):776-785

9. Muskiet MHA, Smits MM, Morsink LM, Diamant M (2014) The gut-renal axis: do incretin-based agents confer renoprotection in diabetes? Nat Rev Nephrol 10(2):88-103

10. Lytvyn Y, Bjornstad P, van Raalte DH, Heerspink HL, Cherney DZI (2019) The new biology of diabetic kidney disease-mechanisms and therapeutic implications. Endocr Rev. https://doi. org/10.1210/endrev/bnz010

11. Bjornstad P, Nehus E, El ghormli L, Bacha F, Libman IM, McKay $S$ et al (2018) Insulin sensitivity and diabetic kidney disease in children and adolescents with type 2 diabetes: an observational analysis of data from the TODAY clinical trial. Am J Kidney Dis 71(1):65-74

12. Tonneijck L, Muskiet MHA, Smits MM, van Bommel EJ, Heerspink HJL, van Raalte DH et al (2017) Glomerular hyperfiltration in diabetes: mechanisms, clinical significance, and treatment. J Am Soc Nephrol 28(4):1023-1039

13. Layton AT, Vallon V (2018) Renal tubular solute transport and oxygen consumption. Curr Opin Nephrol Hypertens. 27(5):384-389

14. Murea M, Freedman BI, Parks JS, Antinozzi PA, Elbein SC, Ma L (2010) Lipotoxicity in diabetic nephropathy: the potential role of fatty acid oxidation. Clin J Am Soc Nephrol 5(12):2373-2379

15. Fine LG, Norman JT (2008) Chronic hypoxia as a mechanism of progression of chronic kidney diseases: from hypothesis to novel therapeutics. Kidney Int 74(7):867-872

16. Espinel E, Agraz I, Ibernon M, Ramos N, Fort J, Serón D (2015) Renal biopsy in type 2 diabetic patients. J Clin Med. 4(5):998-1009

17. Nauck MA, Meier JJ (2018) Incretin hormones: their role in health and disease. Diabetes Obes Metab 20:5-21

18. Drucker DJ, Nauck MA (2006) The incretin system: glucagon-like peptide-1 receptor agonists and dipeptidyl peptidase-4 inhibitors in type 2 diabetes. Lancet 368(9548):1696-1705

19. Muskiet MHA, Tonneijck L, Smits MM, Van Baar MJB, Kramer MHH, Hoorn EJ et al (2017) GLP-1 and the kidney: From physiology to pharmacology and outcomes in diabetes. Nat Rev Nephrol 13:605

20. Skov J (2014) Effects of GLP-1 in the Kidney. Rev Endocr Metab Disord 15(3): 197-207

21. Asmar A, Simonsen L, Asmar M, Madsbad S, Holst JJ, Frandsen E et al (2015) Renal extraction and acute effects of glucagon-like peptide- 1 on central and renal hemodynamics in healthy men. Am J Physiol Metab 308(8):E641-E649

22. Rm C (1978) Evidence for a splanchnic sodium input monitor regulating renal sodium excretion in man lack of dependence upon aldosterone. Circ Res 43:19-23

23. Carraro-Lacroix LR, Malnic G, Girardi ACC (2009) Regulation of $\mathrm{Na}+/ \mathrm{H}+$ exchanger NHE3 by glucagon-like peptide 1 receptor agonist exendin-4 in renal proximal tubule cells. Am J Physiol Physiol 297(6):F1647-F1655

24. Meier JJ (2012) GLP-1 receptor agonists for individualized treatment of type 2 diabetes mellitus. Nat Rev Endocrinol 8(12):728-742

25. Davies MJ, Bain SC, Atkin SL, Rossing P, Scott D, Shamkhalova MS et al (2015) Efficacy and safety of liraglutide versus placebo as add-on to glucose-lowering therapy in patients with type 2 diabetes and moderate renal impairment (LIRA-RENAL): a randomized clinical trial. Diabetes Care 17:142883. https://doi. org/10.2337/dc14-2883

26. Tamborlane WV, Barrientos-Pérez M, Fainberg U, Frimer-Larsen H, Hafez M, Hale PM et al (2019) Liraglutide in children and adolescents with type 2 diabetes. N Engl J Med 381(7):637-646

27. Pfeffer MA, Claggett B, Diaz R, Dickstein K, Gerstein HC, Køber LV et al (2015) Lixisenatide in patients with type 2 diabetes and acute coronary syndrome. N Engl J Med 373(23):2247-2257

28. Muskiet MHA, Tonneijck L, Huang Y, Liu M, Saremi A, Heerspink HJL et al (2018) Lixisenatide and renal outcomes in patients with type 2 diabetes and acute coronary syndrome: an exploratory analysis of the ELIXA randomised, placebo-controlled trial. Lancet Diabetes Endocrinol 6(11):859-869

29. Mann JFE, Ørsted DD, Brown-Frandsen K, Marso SP, Poulter NR, Rasmussen $S$ et al (2017) Liraglutide and renal outcomes in type 2 diabetes. N Engl J Med 377:839-848

30. Husain M, Birkenfeld AL, Donsmark M, Dungan K, Eliaschewitz FG, Franco DR et al (2019) Oral semaglutide and cardiovascular outcomes in patients with type 2 diabetes. $\mathrm{N}$ Engl J Med 381(9):841-851

31. Bethel MA, Mentz RJ, Merrill P, Buse JB, Chan JC, Goodman SG et al (2018) Renal outcomes in the EXenatide study of cardiovascular event lowering (EXSCEL). Diabetes 67(Supplement 1):522

32. Gerstein HC, Colhoun HM, Dagenais GR, Diaz R, Lakshmanan M, Pais P et al (2019) Dulaglutide and renal outcomes in type 2 diabetes: an exploratory analysis of the REWIND randomised, placebo-controlled trial. Lancet 394:131-138

33. Tuttle KR, Lakshmanan MC, Rayner B, Busch RS, Zimmermann AG, Woodward DB et al (2018) Dulaglutide versus insulin glargine in patients with type 2 diabetes and moderate-to-severe chronic kidney disease (AWARD-7): a multicentre, open-label, randomised trial. Lancet Diabetes Endocrinol 6:605-617

34. Mosenzon O, Blicher TM, Rosenlund S, Eriksson JW, Heller S, Hels OH et al (2019) Efficacy and safety of oral semaglutide in patients with type 2 diabetes and moderate renal impairment (PIONEER 5): a placebo-controlled, randomised, phase $3 \mathrm{a}$ trial. Lancet Diabetes Endocrinol 7(7):515-527

35. Perkovic V, Heerspink HL, Chalmers J, Woodward M, Jun M, $\mathrm{Li} Q$ et al (2013) Intensive glucose control improves kidney outcomes in patients with type 2 diabetes. Kidney Int 83:517-523

36. Lewis EJ, Hunsicker LG, Clarke WR, Berl T, Pohl MA, Lewis JB et al (2001) Renoprotective effect of the angiotensin-receptor antagonist irbesartan in patients with nephropathy due to type 2 diabetes. N Engl J Med 345(12):851-860

37. Brenner BM, Cooper ME, de Zeeuw D, Keane WF, Mitch WE, Parving H-H et al (2001Effects) Effects of losartan on renal and cardiovascular outcomes in patients with type 2 diabetes and nephropathy. N Engl J Med 345(12):861-869

38. Moorhead JF, Brunton C, Varghese Z (1997) Glomerular atherosclerosis. Miner Electrolyte Metab 23:287-290

39. Marcovecchio ML, Chiesa ST, Bond S, Daneman D, Dawson S, Donaghue KC et al (2017) ACE inhibitors and statins in adolescents with type 1 diabetes. N Engl J Med 377(18):1733-1745

40. Hung C-C, Tsai J-C, Kuo H-T, Chang J-M, Hwang S-J, Chen H-C (2013) Dyslipoproteinemia and impairment of renal function in diabetic kidney disease: an analysis of animal 
studies, observational studies, and clinical trials. Rev Diabet Stud 10(2-3):110-120

41. Bunck MC, Cornér A, Eliasson B, Heine RJ, Shaginian RM, Wu $Y$ et al (2010) One-year treatment with exenatide vs. insulin glargine: effects on postprandial glycemia, lipid profiles, and oxidative stress. Atherosclerosis 212(1):223-229

42. Tsuboi N, Okabayashi Y, Shimizu A, Yokoo T (2017) The renal pathology of obesity. Kidney Int Rep 2:251-260

43. Foster MC, Hwang S-J, Porter SA, Massaro JM, Hoffmann U, Fox CS (2011) Fatty kidney, hypertension, and chronic kidney disease. Hypertension 58(5):784-790

44. Spit KA, Muskiet MHA, Tonneijck L, Smits MM, Kramer MHH, Joles JA et al (2019) Renal sinus fat and renal hemodynamics: a cross-sectional analysis. Magn Reson Mater Physics Biol Med. https://doi.org/10.1007/s10334-019-00773-z

45. Bahnson JL, Knowler WC, Bantle JP, Bertoni AG, Bray GA, Chen $\mathrm{H}$ et al (2014) Effect of a long-term behavioural weight loss intervention on nephropathy in overweight or obese adults with type 2 diabetes: a secondary analysis of the Look AHEAD randomised clinical trial. Lancet Diabetes Endocrinol 2(10):801-809

46. Li K, Zou J, Ye Z, Di J, Han X, Zhang H et al (2016) Effects of bariatric surgery on renal function in obese patients: a systematic review and meta analysis. PLoS ONE 11(10):e0163907

47. Bjornstad P, Hughan KS, Kelsey MM, Shah A, Lynch JL, Nehus E et al (2019) 123-OR: effect of surgical vs medical therapy on diabetic kidney disease (DKD) in severely obese adolescents with type 2 diabetes (T2D). Diabetes 68(Supplement 1):123-OR

48. Wang C, Li L, Liu S, Liao G, Li L, Chen Y et al (2018) GLP-1 receptor agonist ameliorates obesity-induced chronic kidney injury via restoring renal metabolism homeostasis. PLoS ONE 13(3): $\mathrm{e} 0193473$

49. Strazzullo P, D’Elia L, Kandala N-B, Cappuccio FP (2009) Salt intake, stroke, and cardiovascular disease: meta-analysis of prospective studies. BMJ 339(nov24 1):b4567

50. Titze J (2015) A different view on sodium balance. Curr Opin Nephrol Hypertens 24(1):14-20

51. Muskiet MHA, Tonneijck L, Smits MM, Kramer MHH, Diamant M, Joles JA et al (2016) Acute renal haemodynamic effects of glucagon-like peptide-1 receptor agonist exenatide in healthy overweight men. Diabetes Obes Metab 18(2):178-185

52. Tonneijck L, Smits MM, Muskiet MHA, Hoekstra T, Kramer MHH, Danser AHJ et al (2016) Acute renal effects of the GLP-1 receptor agonist exenatide in overweight type 2 diabetes patients: a randomised, double-blind, placebo-controlled trial. Diabetologia 59(7):1412-1421

53. Tonneijck L, Muskiet MHA, Smits MM, Hoekstra T, Kramer MHH, Danser AHJ et al (2017) Postprandial renal haemodynamic effect of lixisenatide vs once-daily insulin-glulisine in patients with type 2 diabetes on insulin-glargine: an 8-week, randomised, open-label trial. Diabetes Obes Metab 19:1669-1680

54. Tonneijck L, Smits MM, Muskiet MHA, Hoekstra T, Kramer MHH, Danser AHJ et al (2016) Renal effects of DPP-4 inhibitor sitagliptin or GLP-1 receptor agonist liraglutide in overweight patients with type 2 diabetes: a 12-week, randomized, doubleblind, placebo-controlled trial. Diabetes Care 39:2042-2050

55. Inzucchi SE, Zinman B, Fitchett D, Wanner C, Ferrannini E, Schumacher $\mathrm{M}$ et al (2018) How does empagliflozin reduce cardiovascular mortality? Insights from a mediation analysis of the empa-reg outcome trial. Diabetes Care 41(2):356-363

56. Karg MV, Bosch A, Kannenkeril D, Striepe K, Ott C, Schneider MP et al (2018) SGLT-2-inhibition with dapagliflozin reduces tissue sodium content: a randomised controlled trial. Cardiovasc Diabetol 17(1):5

57. Denic A, Mathew J, Lerman LO, Lieske JC, Larson JJ, Alexander MP et al (2017) Single-nephron glomerular filtration rate in healthy adults. N Engl J Med 376(24):2349-2357

58. Cherney DZI, Perkins BA, Soleymanlou N, Maione M, Lai V, Lee A et al (2014) Renal hemodynamic effect of sodium-glucose cotransporter 2 inhibition in patients with type 1 diabetes mellitus. Circulation 129(5):587-597

59. van Bommel EJ, Muskiet MH, van Baar MJ, Tonneijck L, Smits MM, Emanuel AL et al (2019) The renal hemodynamic effects of the SGLT2 inhibitor dapagliflozin are caused by post-glomerular vasodilatation rather than pre-glomerular vasoconstriction in metformin-treated patients with type 2 diabetes in the randomized, double-blind RED trial. Kidney Int 97:202-212

60. Gutzwiller J-P, Tschopp S, Bock A, Zehnder CE, Huber AR, Kreyenbuehl $\mathrm{M}$ et al (2004) Glucagon-like peptide 1 induces natriuresis in healthy subjects and in insulin-resistant obese men. J Clin Endocrinol Metab 89(6):3055-3061

61. von Scholten BJ, Lajer M, Goetze JP, Persson F, Rossing P (2015) Time course and mechanisms of the anti-hypertensive and renal effects of liraglutide treatment. Diabet Med 32(3):343-352

62. Hickey FB, Martin F (2018) Role of the immune system in diabetic kidney disease. Curr Diab Rep 18(4):20

63. Bisgaard LS, Bosteen MH, Fink LN, Sørensen CM, Rosendahl A, Mogensen CK et al (2016) Liraglutide reduces both atherosclerosis and kidney inflammation in moderately uremic LDLr-/Mice. PLoS ONE 11(12):e0168396

Publisher's Note Springer Nature remains neutral with regard to jurisdictional claims in published maps and institutional affiliations. 